\title{
Perfil de diagnósticos de enfermagem CIPE® para pré-natal, por trimestre gestacional
}

ICNP ${ }^{\circledR}$ nursing diagnoses profile for prenatal by gestational trimester Perfil de diagnósticos de enfermería CIPE® para atención prenatal, por trimestre gestacional

Érika Ribeiro Costa ${ }^{1}$ i https://orcid.org//0000-0001-7440-0670

Mariana Miranda Pina ${ }^{2}$ (b) https://orcid. org/0000-0003-0569-485X

Rodrigo Jensen ${ }^{3}$ if https://orcid.org/0000-0001-6191-2001

Milena Temer Jamas ${ }^{3}$ if https://orcid.org/0000-0002-9548-7629

Cristina Maria Garcia de Lima Parada ${ }^{3}$ io https://orcid.org//0000-0002-9597-3635

Como citar: Costa ER, Pina MM, Jensen R, Jamas MT, Parada CM. Perfil de diagnósticos de enfermagem CIPE $®$ para pré-natal, por trimestre gestacional. Acta Paul Enferm. 2021;34:eAPE00575.

DOI

http://dx.doi.org/10.37689/actaape/2021A000575

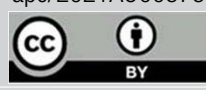

Descritores

Terminologia padronizada em enfermagem: Diagnóstico de enfermagem; Processo de enfermagem; Cuidado pré-natal; Atenção primária à saúde

Keywords

Standardized nursing terminology; Nursing diagnosis; Nursing process; Prenatal care; Primary health care

Descriptores

Terminología normalizada de enfermería Diagnóstico de enfermería; Processo de enfermería; Atención prenatal; Atención primaria de salud

Submetido 25 de Março de 2020

Aceito

2 de Dezembro de 2020

Autor correspondente Cristina Maria Garcia de Lima Parada E-mail: cristina.parada@unesp.br

\section{Resumo}

Objetivo: Identificar diagnósticos de enfermagem pela Classificação Internacional para a Prática de Enfermagem $\left(\mathrm{CIPE}^{\circledR}\right)$ à consulta de enfermagem pré-natal na atenção primária, segundo trimestre gestacional.

Métodos: Estudo transversal, conduzido em uma Unidade de Saúde da Família do município de Botucatu/ SP. Participaram 48 gestantes, atendidas nos meses de agosto a novembro de 2015, nas 95 consultas de enfermagem de pré-natal realizadas. Adotou-se como referencial a Teoria das Necessidades Humanas Básicas.

Resultados: Foram identificados 452 diagnósticos, agrupados em diagnósticos de promoção à saúde, de risco e com foco no problema. A maior parte deles voltaram-se às necessidades psicobiológicas envolvendo necessidades de nutrição, hidratação, eliminações e exercícios e atividades físicas. Evidenciaram-se semelhanças quando considerados os três trimestres de gravidez, o que pode decorrer do fato de terem sido incluídas no estudo apenas gestantes de risco habitual, de forma que frequentemente os diagnósticos propostos guardavam relação com alterações fisiológicas decorrentes da gravidez normal. Foi pequena a proporção de diagnósticos voltados às necessidades psicossociais, com destaque às necessidades de segurança, gregária e aceitação. Nenhum diagnóstico foi proposto relacionado às necessidades psicoespirituais.

Conclusão: A maior parte do conjunto de diagnósticos propostos está no contexto do desenvolvimento de hábitos de vida saudáveis. Porém, nesse processo, há que se considerar a necessidade de ampliar a abordagem da gestante, de forma a contemplar diagnósticos psicossociais e psicoespirituais.

\section{Abstract}

Objective: To identify nursing diagnoses by the International Classification for Nursing Practice (ICNP $\left.{ }^{\circledR}\right)$ to prenatal nursing consultation in primary care according to gestational trimester.

Methods: This is a cross-sectional study conducted at a Family Health Unit in the city of Botucatu/SP. Fortyeight pregnant women, assisted from August to November 2015, participated in the 95 prenatal nursing consultations held. The Theory of Basic Human Needs was adopted as a framework.

Results: We identified 452 diagnoses, grouped into health promotion, risk diagnoses and focused on the problem. Most of them turned to psychobiological needs involving needs for nutrition, hydration, eliminations, and exercises and physical activities. Similarities were evident when considering the three trimesters of pregnancy, which may be due to the fact that only pregnant women of habitual risk were included in the study so that the proposed diagnoses were often related to physiological changes resulting from normal pregnancy. There was a small proportion of diagnoses aimed at psychosocial needs, with emphasis on the needs of security, gregariousness, and acceptance. No diagnosis has been proposed related to psychospiritual needs.

Associação de Assistência à Criança Deficiente, Poços de Caldas, SP, Brasil.

¿Santa Casa de Misericórdia de Guaratinguetá, Guaratinguetá, SP, Brasil.

${ }^{3}$ Faculdade de Medicina de Botucatu, Universidade Estadual Paulista "Júlio de Mesquita Filho", Botucatu, SP, Brasil.

Conflitos de interesse: nada a declarar. 
Conclusion: Most of the set of diagnoses proposed is in the context of developing healthy lifestyle habits. However, in this process, it is necessary to consider the need to expand the approach of pregnant women, in order to include psychosocial and psychospiritual diagnoses.

\section{Resumen}

Objetivo: Identificar diagnósticos de enfermería a través de la Clasificación Internacional de la Práctica de Enfermería (CIPE $\left.{ }^{\circledR}\right)$ para consultas de enfermería prenatal en la atención primaria, según el trimestre gestacional.

Métodos: Estudio transversal, conducido en una Unidad de Salud de la Familia en el municipio de Botucatu, estado de São Paulo. Participaron 48 mujeres embarazadas, atendidas entre los meses de agosto y noviembre de 2015, en 95 consultas de enfermería prenatal. Se adoptó la teoría de las necesidades humanas básicas como marco referencial.

Resultados: Se identificaron 452 diagnósticos, agrupados en diagnósticos de promoción de la salud, de riesgo y con foco en el problema. La mayor parte se relacionó con las necesidades psicobiológicas, que incluye necesidades de nutrición, hidratación, eliminación y ejercicio y actividad física. Se observaron semejanzas cuando se consideraron los tres trimestres del embarazo, lo que puede provenir del hecho de haber incluido solo gestantes de riesgo normal en el estudio. De esta forma, los diagnósticos propuestos estaban con frecuencia asociados con alteraciones fisiológicas resultantes de un embarazo normal. La proporción de diagnósticos relacionados con las necesidades psicosociales fue pequeña, con énfasis en la necesidad de seguridad, gregaria y de aprobación. No se propuso ningún diagnóstico relacionado con las necesidades psicoespirituales.

Conclusión: La mayor parte del conjunto de diagnósticos propuestos está dentro del contexto del desarrollo de hábitos de vida más saludables. Sin embargo, en este proceso hay que considerar la necesidad de ampliar el enfoque hacia la mujer embarazada, a fin de contemplar diagnósticos psicosociales y psicoespirituales.

\section{Introdução}

O Processo de Enfermagem (PE) organiza e qualifica o trabalho do enfermeiro, na medida em que confere especificidade e qualidade ao cuidado de enfermagem. Como método de trabalho, este permite acompanhar a evolução clínica dos indivíduos assistidos, direciona o cuidado e facilita o registro das atividades, amparando em respaldo legal a equipe de enfermagem. ${ }^{(1,2)}$

O PE, que na atenção primária corresponde à Consulta de Enfermagem, é organizado por etapas inter-relacionadas e interdependentes, a saber: Coleta de Dados ou Histórico de Enfermagem, Diagnóstico de Enfermagem (DE), Planejamento de Enfermagem, Implementação das Ações e Avaliação de Enfermagem. Para sua operacionalização, obrigatória nos locais em que ocorre atendimento de enfermagem, o enfermeiro deve lançar máo dos sistemas de linguagem padronizada e pautar-se em referenciais teóricos. ${ }^{(3)}$

A Classificação Internacional para a Prática de Enfermagem (CIPE $)$ constitui instrumento de informaçáo para descrever a assistência de enfermagem. Seus termos proporcionam documentação padronizada do cuidado prestado ao usuário. Assim, provê dados que evidenciam a contribuição da enfermagem na atenção à saúde e promove avanços na prática profissional, por meio de sua aplicação na assistência, educação, pesquisa e gestão em en- fermagem. É uma terminologia combinatória e seu modelo de sete eixos (foco, julgamento, meios, ação, localização, tempo e cliente) permite a composição de afirmativas de diagnósticos, intervenções e resultados de enfermagem.

$\mathrm{Na} \mathrm{CIPE}^{\circ}$, o DE é definido como um título atribuído pelo enfermeiro ao tomar decisão sobre um fenômeno que é foco de intervençôes de enfermagem. O diagnóstico é constituído pela composição de termo relacionado a um foco e um julgamento. Em casos específicos, não é obrigatório o termo relacionado a julgamento, quando se trata de um achado clínico. ${ }^{(4,5)}$

Há experiências de utilização da $\mathrm{CIPE}^{\circ}$ associada à Teoria de Enfermagem de Wanda Horta. ${ }^{(6,7)}$ Tal teoria partiu das Necessidades Humanas Básicas, que foram categorizadas por Maslow de forma hierárquica, de menor à maior importância. Horta, entretanto, classificou as necessidades em duas categorias: psicobiológicas e psicossociais. Tinha por pressuposto que ao identificar as necessidades na primeira etapa do PE, o Histórico de Enfermagem, o enfermeiro estaria apto a definir as necessidades de saúde do usuário e a elaborar um plano assistencial; neste deveria constar o grau de dependência do paciente e as intervençóes a serem realizadas. ${ }^{(8)}$

$\mathrm{O}$ presente estudo, voltado à aplicação da $\mathrm{CIPE}^{\circ}$ na assistência de enfermagem no pré-natal, justifica-se pelas potencialidades de uso desse sistema de classificação na atenção primária e por serem os es- 
tudos de prevalência de diagnósticos de enfermagem em gestantes ainda escassos. Estudo de revisão que abordou as publicaçóes relacionadas à $\mathrm{CIPE}^{\circ}$ entre 2008 e 2017 identificou 35 trabalhos, dos quais apenas um artigo internacional voltava-se a gestantes em acompanhamento pré-natal. ${ }^{(9)}$ Justifica-se, também, por serem as mulheres as maiores usuárias do Sistema Único de Saúde (SUS) e pelo fato de a principal política de saúde voltada a esse grupo ser a Rede Cegonha, que tem entre suas diretrizes a ampliação do acesso e a qualificação da assistência pré-natal. ${ }^{(10)}$ Por fim, justifica-se pela possibilidade de instrumentalizar os enfermeiros no cumprimento da Resolução 358/2009 do Conselho Federal de Enfermagem, que dispóe sobre a obrigatoriedade da realização do PE. ${ }^{(3)}$

Assim, objetivou-se identificar diagnósticos de enfermagem da CIPE ${ }^{\circ}$ na consulta de enfermagem pré-natal na atenção primária, segundo o trimestre gestacional.

\section{Métodos}

Trata-se de estudo transversal, conduzido em uma Unidade de Saúde da Família, localizada na periferia do município de Botucatu/SP. Nessa Unidade são atendidas apenas gestantes de risco habitual.

Adotou-se como referencial a Teoria das Necessidades Humanas Básicas, ${ }^{(8)}$ muito embora se reconheça que as necessidades de saúde são impostas pela vida social e, por isso, não se restringem àquelas referidas no âmbito dos aspectos biopsicossociais. ${ }^{(6)}$

A população do estudo foi constituída por todas as gestantes atendidas em consulta de pré-natal (agendadas ou eventuais) nos meses de agosto a novembro de 2015, visto não ter havido recusa para participação na pesquisa. Assim, participaram 48 mulheres, totalizando 95 consultas de enfermagem de pré-natal realizadas no período.

A coleta de dados foi conduzida por uma docente da área de obstetrícia, uma graduanda em enfermagem e duas enfermeiras. As consultas aconteceram conforme a rotina da Unidade, em ambiente propício. Ao término da consulta, após a gestante deixar a sala de atendimento, instrumento cons- truído para este estudo era preenchido, contendo os dados de identificação da gestante, a descrição da história clínica e os diagnósticos de enfermagem.

As variáveis para caracterização das participantes do estudo foram: idade (anos), naturalidade (cidade de nascimento), situação conjugal (com companheiro, sem companheiro), aprovação escolar (anos), história obstétrica: número de gestaçôes, partos, abortos, cesáreas e número de filhos vivos.

Para a elaboração dos diagnósticos foram seguidos quatro passos: 1- leitura do histórico de enfermagem, a identificar palavras significativas; 2- releitura das palavras destacadas, organizando-as, tomando por base o raciocínio clínico; 3- seleção de títulos de diagnósticos de enfermagem da CIPE versão 2015 a partir de conceitos pré-coordenados; ${ }^{(4)}$ e 4- construção dos títulos, utilizando conceitos primitivos da $\mathrm{CIPE}^{\circ}$ versão 2015, seguindo a ISO 18104:2014. (5) Os diagnósticos foram organizados em promoção da saúde, de risco e com foco no problema. Posteriormente, estes diagnósticos foram atualizados à CIPE versão 2019-2020. ${ }^{(11)}$

Para análise de dados construiu-se um banco de dados em planilha no software Excel, sendo os dados submetidos à análise descritiva.

O estudo foi aprovado pelo Comitê de Ética em Pesquisa da Faculdade de Medicina de Botucatu da Universidade Estadual Paulista Júlio de Mesquita Filho (CAAE: 44875415.0.0000.5411) e seguiu as recomendaçóes de pesquisas envolvendo seres humanos.

\section{Resultados}

A caracterização das gestantes indica tratarem-se de mulheres jovens, com média de idade de 24,4 anos $(\mathrm{DP}=7,83)$. A maioria tinha oito ou mais anos de aprovação escolar $(73,5 \%)$; era natural do município $(61,8 \%)$; tinha companheiro $(73,5 \%)$; não era primigesta $(73,5 \%)$ e não tinha história de aborto (70,6\%). No total, 452 diagnósticos de enfermagem foram identificados nas 95 consultas de enfermagem, média aproximada de cinco diagnósticos por consulta. Na tabela 1 são apresentas as necessidades psicobiológicas, sendo contemplados diagnósticos de promoção da saúde, de risco e com foco no problema. 
Tabela 1. Diagnósticos de enfermagem de promoção da saúde, de risco e com foco no problema, segundo trimestre de gestação e necessidades psicobiológicas, apresentados por Necessidades Humanas Básicas ( $\mathrm{n}=95)$

\begin{tabular}{|c|c|c|c|}
\hline Diagnósticos de enfermagem & $\begin{array}{c}1^{0} \\
\text { trimestre } \\
n(\%)\end{array}$ & $\begin{array}{c}2^{0} \\
\text { trimestre } \\
n(\%)\end{array}$ & $\begin{array}{c}3^{0} \\
\text { trimestre } \\
n(\%)\end{array}$ \\
\hline \multicolumn{4}{|l|}{ Diagnósticos de promoção da saúde (código) } \\
\hline \multicolumn{4}{|l|}{ Hidratação } \\
\hline Hidratação, adequada (10042065) & $2(5,7)$ & $4(10,5)$ & $4(18,2)$ \\
\hline \multicolumn{4}{|l|}{ Nutrição } \\
\hline Adesão ao regime dietético (10030159) & $8(22,9)$ & $8(21,1)$ & $5(22,8)$ \\
\hline Peso, eficaz (10027385) & $0(0,0)$ & $0(0,0)$ & $1(4,5)$ \\
\hline \multicolumn{4}{|l|}{ Eliminações } \\
\hline Promover eliminação intestinal, eficaz (10036717) & $0(0,0)$ & $0(0,0)$ & $2(9,1)$ \\
\hline \multicolumn{4}{|l|}{ Sono/repouso } \\
\hline Sono, adequado (10024930) & $0(0,0)$ & $1(2,6)$ & $0(0,0)$ \\
\hline \multicolumn{4}{|l|}{ Exercício e atividade física } \\
\hline Adesão ao regime de exercício físico (10030163) & $1(2,8)$ & $2(5,3)$ & $1(4,5)$ \\
\hline \multicolumn{4}{|l|}{ Terapêutica } \\
\hline Adesão ao regime terapêutico (10030205) & $2(5,7)$ & $5(13,1)$ & $0(0,0)$ \\
\hline Resultado laboratorial, negativo* & $8(22,9)$ & $2(5,3)$ & $1(4,5)$ \\
\hline \multicolumn{4}{|l|}{ Reprodução } \\
\hline $\begin{array}{l}\text { Complicação durante a gestação (gravidez), ausente } \\
\text { (10042446) }\end{array}$ & $14(40,0)$ & $16(42,1)$ & $8(36,4)$ \\
\hline \multicolumn{4}{|l|}{ Diagnósticos de risco (código) } \\
\hline \multicolumn{4}{|l|}{ Oxigenação } \\
\hline Risco de dispneia* & $0(0,0)$ & $0(0,0)$ & $1(6,2)$ \\
\hline \multicolumn{4}{|l|}{ Nutrição } \\
\hline $\begin{array}{l}\text { Risco de estar com peso abaixo do esperado } \\
\text { (10037586) }\end{array}$ & $0(0,0)$ & $2(8,3)$ & $0(0,0)$ \\
\hline $\begin{array}{l}\text { Risco de ingestão de alimentos, insuficiente (ou } \\
\text { deficitária) (10023021) }\end{array}$ & $1(10,0)$ & $0(0,0)$ & $2(12,5)$ \\
\hline $\begin{array}{l}\text { Risco de ingestão de alimentos, excessiva } \\
\text { (10015114) }\end{array}$ & $0(0,0)$ & $5(20,8)$ & $4(25,0)$ \\
\hline \multicolumn{4}{|l|}{ Eliminações } \\
\hline Risco de constipação (10015053) & $5(50,0)$ & $9(37,5)$ & $4(25,0)$ \\
\hline $\begin{array}{l}\text { Risco de incontinência urinária, de urgência } \\
\text { (10026848) }\end{array}$ & $0(0,0)$ & $1(4,2)$ & $0(0,0)$ \\
\hline \multicolumn{4}{|l|}{ Terapêutica } \\
\hline Risco de doença: diabetes* & $0(0,0)$ & $1(4,2)$ & $1(6,2)$ \\
\hline Risco de infecção urinária (10051950) & $0(0,0)$ & $3(12,5)$ & $1(6,2)$ \\
\hline \multicolumn{4}{|l|}{ Reprodução } \\
\hline Risco de abortamento, espontâneo* & $3(30,0)$ & $0(0,0)$ & $1(6,2)$ \\
\hline $\begin{array}{l}\text { Risco de complicações durante a gestação } \\
\text { (gravidez): hipertensão* }\end{array}$ & $1(10,0)$ & $3(12,5)$ & $1(6,2)$ \\
\hline $\begin{array}{l}\text { Risco de complicações durante a gestação } \\
\text { (gravidez): anemia* }\end{array}$ & $0(0,0)$ & $0(0,0)$ & $1(6,2)$ \\
\hline \multicolumn{4}{|l|}{ Diagnósticos com foco no problema (código) } \\
\hline \multicolumn{4}{|l|}{ Hidratação } \\
\hline Ingestão de líquidos, prejudicada (10029873) & $17(17,2)$ & $18(17,8)$ & $10(14,1)$ \\
\hline \multicolumn{4}{|l|}{ Nutrição } \\
\hline Ingestão nutricional, prejudicada (10023009) & $22(22,3)$ & $21(20,8)$ & $19(26,8)$ \\
\hline Ingestão de alimentos, excessiva (10000682) & $0(0,0)$ & $1(1,0)$ & $1(1,4)$ \\
\hline Sobrepeso (10027300) & $1(1,0)$ & $1(1,0)$ & $1(1,4)$ \\
\hline Obesidade (10013457) & $0(0,0)$ & $2(2,0)$ & $1(1,4)$ \\
\hline Náusea (10013457) & $18(18,2)$ & $8(7,9)$ & $3(4,2)$ \\
\hline Vômito (10025981) & $9(9,1)$ & $4(3,9)$ & $1(1,4)$ \\
\hline \multicolumn{4}{|l|}{ Eliminaç̧ões } \\
\hline Constipação (10000567) & $5(5,0)$ & $4(3,9)$ & $2(2,9)$ \\
\hline Descarga (ou fluxo) vaginal, excessiva (10008688) & $2(2,0)$ & $3(3,0)$ & $3(4,2)$ \\
\hline \multicolumn{4}{|l|}{ Sono/repouso } \\
\hline Sono, prejudicado (10027226) & $0(0,0)$ & $2(2,0)$ & $10(14,1)$ \\
\hline
\end{tabular}

\begin{tabular}{|c|c|c|c|}
\hline Diagnósticos de enfermagem & $\begin{array}{c}1^{\circ} \\
\text { trimestre } \\
n(\%)\end{array}$ & $\begin{array}{c}2^{\circ} \\
\text { trimestre } \\
\mathrm{n}(\%)\end{array}$ & $\begin{array}{c}3^{\circ} \\
\text { trimestre } \\
n(\%)\end{array}$ \\
\hline \multicolumn{4}{|l|}{ Exercício e atividade física } \\
\hline $\begin{array}{l}\text { Não adesão ao regime de exercício físico } \\
\text { (10022657) }\end{array}$ & $10(10,1)$ & $6(6,0)$ & $1(1,4)$ \\
\hline \multicolumn{4}{|l|}{ Cuidado corporal } \\
\hline Déficit de autocuidado (10023410) & $0(0,0)$ & $2(2,0)$ & $0(0,0)$ \\
\hline \multicolumn{4}{|l|}{ Integridade cutâneo mucosa } \\
\hline Integridade da pele, prejudicada (10001290) & $1(1,0)$ & $4(3,9)$ & $0(0,0)$ \\
\hline \multicolumn{4}{|l|}{ Regulação vascular } \\
\hline Edema periférico (10027482) & $0(0,0)$ & $0(0,0)$ & $1(1,4)$ \\
\hline \multicolumn{4}{|l|}{ Regulação imunológica } \\
\hline Não adesão a regime de imunização (10030026) & $1(1,0)$ & $3(3,0)$ & $2(2,9)$ \\
\hline \multicolumn{4}{|l|}{ Percepção } \\
\hline Dor de cabeça* & $2(2,0)$ & $4(3,9)$ & $0(0,0)$ \\
\hline Dor na região pubiana* & $4(4,1)$ & $8(7,9)$ & $3(4,2)$ \\
\hline Dor de falso trabalho de parto (10007549) & $0(0,0)$ & $0(0,0)$ & $3(4,2)$ \\
\hline Controle da dor (10025831) & $0(0,0)$ & $1(1,0)$ & $0(0,0)$ \\
\hline Controle da dor, inadequado (10039910) & $0(0,0)$ & $2(2,0)$ & $0(0,0)$ \\
\hline Cólica no útero* & $2(2,0)$ & $0(0,0)$ & $1(1,4)$ \\
\hline Prurido na vagina* & $1(1,0)$ & $0(0,0)$ & $1(1,4)$ \\
\hline \multicolumn{4}{|l|}{ Ambiente } \\
\hline Dependência de drogas (10041381) & $1(1,0)$ & $1(1,0)$ & $0(0,0)$ \\
\hline Abuso de tabaco (ou de fumo) (10022247) & $2(2,0)$ & $3(3,0)$ & $4(5,6)$ \\
\hline \multicolumn{4}{|l|}{ Terapêutica } \\
\hline Não adesão ao regime terapêutico (10022155) & $1(1,0)$ & $3(3,0)$ & $4(5,6)$ \\
\hline
\end{tabular}

*Diagnóstico construído a partir de termos primitivos

No primeiro trimestre, os diagnósticos de enfermagem de promoção da saúde mais frequentes foram: Complicação durante a Gestação (Gravidez), Ausente (40,0\%), Adesão ao Regime Dietético (22,9\%) e Resultado Laboratorial, Negativo (22,9\%); no segundo trimestre, destacaram-se Complicação durante a Gestação (Gravidez), Ausente $(42,1 \%)$ e Adesão ao Regime Dietético $(21,1 \%)$ e; no terceiro trimestre, os mais frequentes foram Complicação durante a Gestação (Gravidez), Ausente (36,4\%) e Adesão ao Regime Dietético (22,8\%).

Os principais diagnósticos de risco no primeiro trimestre foram: Risco de Constipação (50,0\%) e Risco de Abortamento, Espontâneo (30,0\%); no segundo trimestre destacaram-se Risco de Constipação (37,5\%) e Risco de Ingestáo de Alimentos, Excessiva $(20,8 \%)$ e; no terceiro trimestre houve destaque para Risco de Constipação (25,0\%) e Risco de Ingestão de Alimentos, Excessiva (25,0\%).

No primeiro trimestre, os principais diagnósticos de enfermagem com foco no problema foram: Ingestáo Nutricional, Prejudicada $(22,3 \%)$ e Náusea (18,2\%); no segundo trimestre, destacaram-se Ingestão Nutricional, Prejudicada (20,8\%) 
e Ingestão de Líquidos, Prejudicada (17,8\%) e; no terceiro trimestre, foram frequentes Ingestão Nutricional, Prejudicada (26,8\%), Ingestão de Líquidos, Prejudicada $(14,1 \%)$ e Sono, Prejudicado $(14,1 \%)$.

No âmbito das necessidades psicossociais, os diagnósticos de enfermagem de promoção da saúde, de risco e com foco no problema são apresentados na tabela 2 .

Tabela 2. Diagnósticos de enfermagem de promoção da saúde, de risco e com foco no problema, por trimestre de gestação, segundo necessidades psicossociais, apresentados por Necessidades Humanas Básicas $(\mathrm{n}=36)$

\begin{tabular}{|c|c|c|c|}
\hline Diagnósticos de enfermagem & $\begin{array}{c}1^{0} \\
\text { trimestre } \\
n(\%)\end{array}$ & $\begin{array}{c}2^{0} \\
\text { trimestre } \\
n(\%)\end{array}$ & $\begin{array}{c}3^{\circ} \\
\text { trimestre } \\
n(\%)\end{array}$ \\
\hline \multicolumn{4}{|l|}{ Diagnósticos de promoção da saúde (código) } \\
\hline \multicolumn{4}{|l|}{ Gregária } \\
\hline Apoio familiar, positivo (10045702) & $0(0,0)$ & $2(8,0)$ & $4(50,0)$ \\
\hline \multicolumn{4}{|l|}{ Diagnósticos de risco (código) } \\
\hline \multicolumn{4}{|l|}{ Aceitação } \\
\hline $\begin{array}{l}\text { Risco de gestação (gravidez), não intencional } \\
\text { (10023182) }\end{array}$ & $2(66,7)$ & $7(28,0)$ & $0(0,0)$ \\
\hline \multicolumn{4}{|l|}{ Aprendizagem ou educação em saúde } \\
\hline Regime de cuidado pré-natal, comprometido* & $0(0,0)$ & $4(16,0)$ & $0(0,0)$ \\
\hline \multicolumn{4}{|l|}{ Diagnósticos com foco no problema (código) } \\
\hline \multicolumn{4}{|l|}{ Aprendizagem ou educação em saúde } \\
\hline Falta de conhecimento sobre doença (10021994) & $0(0,0)$ & $1(4,0)$ & $0(0,0)$ \\
\hline Baixo comparecimento escolar (10037777) & $0(0,0)$ & $1(4,0)$ & $0(0,0)$ \\
\hline \multicolumn{4}{|l|}{ Gregária } \\
\hline Falta de apoio familiar (10022473) & $0(0,0)$ & $3(12,0)$ & $1(12,5)$ \\
\hline Atitude familiar, conflituosa (10022456) & $0(0,0)$ & $1(4,0)$ & $0(0,0)$ \\
\hline Falta de apoio social (10022753) & $1(33,3)$ & $0(0,0)$ & $0(0,0)$ \\
\hline \multicolumn{4}{|l|}{ Autoestima } \\
\hline Baixa autoestima, situacional (10000844) & $0(0,0)$ & $1(4,0)$ & $0(0,0)$ \\
\hline \multicolumn{4}{|l|}{ Segurança } \\
\hline Ansiedade (10000477) & $0(0,0)$ & $1(4,0)$ & $2(25,0)$ \\
\hline Ansiedade, reduzida (10027858) & $0(0,0)$ & $1(4,0)$ & $0(0,0)$ \\
\hline Medo (10000703) & $0(0,0)$ & $2(8,0)$ & $1(12,5)$ \\
\hline Medo, reduzido (10027889) & $0(0,0)$ & $1(4,0)$ & $0(0,0)$ \\
\hline
\end{tabular}

* Diagnóstico construído a partir de termos primitivos

Entre os títulos diagnósticos psicossociais relacionados à promoção da saúde, no segundo e terceiro trimestres, foram propostos Apoio Familiar, Positivo, citados duas $(8,0 \%)$ e quatro vezes $(50,0 \%)$, respectivamente. Nenhum diagnóstico psicossocial de promoção da saúde foi proposto no primeiro trimestre (Tabela 2).

Títulos de risco foram descritos no primeiro e segundo trimestres, destacando-se o Risco de Gestação (Gravidez), Não Intencional, citado duas $(66,7 \%)$ e sete vezes $(28,0 \%)$. Entre os títulos diag- nósticos com foco no problema, na área psicossocial, foi proposto no primeiro trimestre apenas Falta de Apoio Social (um caso, 33,3\%); no segundo trimestre, os mais comuns foram Falta de Apoio Familiar (três casos, 12,0\%) e Medo (dois casos, $8,0 \%$ ) e no terceiro trimestre destacou-se a Ansiedade (dois casos, 25,0\%) (Tabela 2).

A tabela 3 é referente ao conjunto de diagnósticos por necessidades humanas básicas.

Tabela 3. Total de títulos diagnósticos segundo necessidades psicobiológicas e psicossociais por trimestre de gestação $(\mathrm{n}=452)$

\begin{tabular}{l|c|c|c}
\hline Diagnósticos & $\begin{array}{c}\mathbf{1}^{\mathbf{0}} \\
\text { trimestre } \\
\mathbf{n}(\%)\end{array}$ & $\begin{array}{c}\mathbf{2}^{\mathbf{0}} \\
\text { trimestre } \\
\mathbf{n}(\%)\end{array}$ & $\begin{array}{c}\mathbf{3}^{\mathbf{0}} \\
\text { trimestre } \\
\mathbf{n}(\%)\end{array}$ \\
\hline $\begin{array}{l}\text { Necessidades psicobiológicas } \\
\quad \text { Promoção da saúde }\end{array}$ & $35(23,8)$ & $38(20,2)$ & $22(18,8)$ \\
Risco & $10(6,8)$ & $24(12,8)$ & $16(13,7)$ \\
Foco no problema & $99(67,3)$ & $101(53,7)$ & $71(60,7)$ \\
Necessidades psicossociais & & & \\
\hline Promoção da saúde & $0(0,0)$ & $2(1,1)$ & $4(3,4)$ \\
Risco & $2(1,4)$ & $11(5,8)$ & $0(0,0)$ \\
\hline Foco no problema & $1(0,7)$ & $12(6,4)$ & $4(3,4)$ \\
\hline
\end{tabular}

As necessidades psicobiológicas foram amplamente identificadas, quando comparadas às psicossociais. Independentemente do trimestre gestacional, as categorias dos títulos diagnósticos mais frequentes, entre as necessidades psicobiológicas, foram: com foco no problema, de promoção da saúde e risco. Considerando-se as necessidades psicossociais, os títulos diagnósticos foram pouco mais frequentes no segundo trimestre gestacional (Tabela 3).

\section{Discussão}

Foi identificada semelhança entre os diagnósticos presentes nos três trimestres. Isto pode decorrer do fato de terem sido incluídas no estudo apenas gestantes de risco habitual, de forma que frequentemente os diagnósticos propostos, independentemente de tratarem-se de diagnósticos de promoção da saúde, risco ou com foco no problema, guardavam relação com alteraçóes fisiológicas decorrentes da gravidez normal.

Merece destaque a pequena proporção de diagnósticos voltados às necessidades psicossociais e 
ausência de diagnósticos relacionados às necessidades psicoespirituais, fato que pode decorrer dos instrumentos de registro utilizados nas consultas de enfermagem: ficha perinatal e caderneta da gestante. Tais instrumentos foram propostos pela Rede Cegonha $^{(10)}$ e, apesar dos avanços proporcionados à proposta do atendimento integral e adoção da clínica ampliada no pré-natal, pouco inclui sobre necessidades psicossociais e nada das necessidades psicoespirituais das gestantes. Tal fato sinaliza a necessidade de maior aproximação de modelos que incluam diferentes necessidades, superando a visão biologicista. A identificação das necessidades psicossociais e psicoespirituais poderá ser viabilizada a partir do uso de um referencial teórico, na consulta de enfermagem, que possibilite e valorize esse olhar ampliado, independentemente do instrumento de registro adotado.

O reconhecimento de que as condiçóes econômicas e sociais influenciam decisivamente as condições de saúde de pessoas e populaçóes e que a maior parte da carga das doenças acontece em decorrência das condiçóes em que as pessoas nascem, vivem, trabalham e envelhecem, ${ }^{(12)}$ pode resultar na ampliação dos títulos diagnósticos relacionados às necessidades psicossociais. Assim, cabe a gestores de municípios promover a discussáo dessa temática com profissionais de saúde, especialmente com os enfermeiros, de forma a adaptar os instrumentos de coleta de dados para que esse olhar possa ser valorizado nas consultas de enfermagem de pré-natal.

Independente do trimestre gestacional, considerando-se os diagnósticos de enfermagem de promoção da saúde e de foco no problema, a necessidade de nutrição destacou-se. Sabe-se ser a gravidez período importante, que pode influenciar o bem-estar das futuras geraçóes ${ }^{(13,14)}$ e que a nutrição na gravidez repercute náo apenas na saúde materna, mas também na saúde fetal e infantil, ${ }^{(14,15)}$ o que torna a prática da alimentação saudável meta a ser alcançada pelas gestantes.

Tendo-se por pressuposto que eventuais riscos devam ser identificados nas consultas pré-natais, aspectos relacionados à alimentação devem ser abordados em todos os contatos com as gestantes, de forma a identificar dificuldade na oferta e no acesso aos alimentos, além da influência que essa alimentação sofre pelos fatores culturais e simbólicos. ${ }^{(16)}$ Assim, justifica-se o achado de elevada frequência de diagnósticos de enfermagem associados à necessidade de nutrição e infere-se a compreensão das gestantes quanto à sua relevância, pela elevada frequência do título Adesão ao Regime Dietético nos três trimestres da gestação. Não sem dificuldade, em contraposição também foi bastante elencado o diagnóstico Ingestão Nutricional, Prejudicada.

O título Ingestão Nutricional, Prejudicada pode estar, de alguma forma, associado ao diagnóstico Náusea, encontrado neste estudo especialmente nos dois primeiros trimestres da gestação. Esse evento, mais comum durante as primeiras semanas de gestação, seis a oito semanas, até a $20^{a}$ semana, pode ocorrer em qualquer hora do dia ou da noite, embora seja mais frequente pela manhã, e afeta $85 \%$ das gestantes, sendo que, na forma mais severa de 0,3 a 3\%. ${ }^{(17)}$ Ainda destaca-se, no contexto da necessidade de nutrição, o diagnóstico de Risco de Ingestão de Alimentos, Excessiva, identificado sempre que observado ganho de peso acima do esperado, calculado a partir do Índice de Massa Corporal (IMC) pré-gestacional e considerando a idade gestacional. Tal diagnóstico foi elencado exclusivamente para o segundo e terceiro trimestre, provavelmente após melhora no quadro de náuseas.

Ingestão de Líquidos, Prejudicada, foi título diagnóstico comum aos três trimestres gestacionais e Hidratação, Adequada, esteve entre os mais comuns no terceiro trimestre de gravidez, quando considerados os diagnósticos de promoção da saúde, condição que também pode estar relacionada à melhora no quadro de náusea anteriormente citada. É conhecido o papel desenvolvido pela água no organismo, com relação à digestão, metabolismo, transporte de nutrientes, manutenção da função vascular e regulação da temperatura. Todavia, o estabelecimento das necessidades de ingesta é complexo, não apenas na gravidez, mas também em outras fazes da vida, devido à diversidade das atividades humanas e a complexidade do processo de regulação da água no corpo humano. Assim, guideline internacional para ingesta adequada de água, proposto por organizaçóes científicas da Europa, 
China, Austrália e Estados Unidos da América, recomenda que gestantes recebam no total $2300 \mathrm{ml}$ de água por dia, incluindo a água dos alimentos, estimada em 20\%. Dessa forma, a ingesta média de água indicada, $1840 \mathrm{ml}$ por dia, é valor próximo ao adotado neste estudo para indicação do título diagnóstico Ingestão de Líquidos, Prejudicada, que é inferior a $2000 \mathrm{ml}$ por dia. Nenhum estudo foi identificado abordando a ingesta hídrica para a população brasileira. No contexto internacional, a Autoridade Europeia para a Segurança Alimentar aponta que entre os países-membro, parte significativa da população ingere menor quantidade de água que a recomendada. ${ }^{(18)}$

Mesmo que indiretamente, o título Ingestão de Líquidos, Prejudicada, associa-se ao título diagnóstico Risco de Constipação, um dos mais frequentes nos três trimestres gestacionais quando considerado o grupo dos diagnósticos de risco. A constipação é um distúrbio intestinal funcional caracterizado por dor e desconforto, esforço, fezes endurecidas e sensação de evacuação incompleta, comum durante a gravidez: uma em cada três gestantes apresentam esse quadro, ${ }^{(19)}$ sendo essa condição mais frequente na gravidez que fora dela e tendo como relevante fator de risco a falta de atividade física, comum durante a gestação. ${ }^{(20)}$ Neste estudo, tratando-se de título diagnóstico de risco, o critério para sua definição tomou por base a frequência de evacuação $\mathrm{e}$, assim, todas as gestantes que referiram intervalo entre as evacuaçóes de dois dias ou mais, tiveram tal diagnóstico apontado, independentemente de outros sinais ou sintomas. No seguimento pré-natal destes casos, houve preocupação em acompanhar a evolução para o diagnóstico com foco no problema, com título de Constipação, o qual também foi identificado nos três trimestres de gestação, mas com menor ocorrência.

Apesar das evidências científicas dos benefícios que a prática de atividade física proporciona, há pouca adesão a ela quando a mulher entra no ciclo gravídico-puerperal. Essa foi condição encontrada na presente investigação, destacadamente no primeiro trimestre da gestação, visto que aproximadamente $10 \%$ dos diagnósticos com foco no problema foi Não Adesão ao Regime de Exercício
Físico. Alguns estudos quantitativos apontam como causa para isso o medo, não justificável, de repercussóes negativas para a gravidez. ${ }^{(21)}$ Ao contrário, resultados favoráveis são identificados quando se consideram aspectos obstétricos e fetais. Assim, os profissionais precisam deixar claro que os benefícios de 150 minutos de atividade física por semana, ao longo da gravidez, superam os riscos para a grande maioria das gestantes e não focar o aconselhamento em contra indicaçóes ou perigos potenciais. As mulheres que já praticam atividade física devem ser orientadas de que manter altos níveis de atividade é seguro e saudável para a mãe e o bebê. ${ }^{(22)}$

Alguns diagnósticos de promoção da saúde, entre os mais frequentes do primeiro e segundo trimestres, foram Resultado Laboratorial, Negativo e Adesão a Regime Terapêutico, respectivamente. Indicam a oportunidade de desenvolvimento de açóes preventivas, bem como educativas, visto que muitas vezes a gravidez é o único momento em que as mulheres são atendidas nos serviços de saúde.

Outros diagnósticos de risco e com foco no problema não permearam todos os trimestres da gravidez, sendo achados em trimestres específicos, caso dos títulos diagnósticos Risco de Abortamento, Espontâneo e Dor de Falso Trabalho de Parto e os diagnósticos de desconforto, como Dor na Região Pubiana e Sono, Prejudicado.

O diagnóstico Dor na Regiāo Pubiana pode decorrer de inúmeras causas, é condição comum e pode afetar negativamente o bem estar da gestante. ${ }^{(23)}$ Durante as consultas pré-natais merece atençáo a referência a essa dor, em geral de difícil diagnóstico, pelos múltiplos fatores de confusão associados, mesmo numa gestação normal, decorrentes das mudanças anatômicas no abdômen e pelve e do crescimento uterino. Destaca-se que a identificação da causa básica e tratamento imediato sempre que necessário, são fundamentais para não colocar em risco a saúde da mãe e do feto. ${ }^{(24)}$ Sua ocorrência deve ser sinal de alerta, podendo estar associada ao risco de abortamento ou trabalho de parto prematuro, a depender da época de ocorrência, primeiro ou terceiro trimestre, respectivamente.

Quanto aos distúrbios do sono, decorrem de alterações hormonais, fisiológicas, metabólicas, psicológicas e posturais relacionadas à gravidez. 
São comuns, podem estar associados a desfechos negativos durante a gravidez e são frequentemente pouco diagnosticados e tratados. ${ }^{(25)}$ Considerandose a possibilidade de minimizar esses sintomas com condutas simples, cabe aos profissionais proporem ações de enfermagem com essa finalidade às gestantes.

$\mathrm{Na}$ área psicossocial, destacou-se a necessidade gregária, em especial com diagnósticos de enfermagem que remetiam ao apoio familiar. A rede social de apoio tem sido enfatizada como aspecto relevante para o bem-estar materno durante a gestação e o nascimento dos filhos, podendo modificar desfechos no peso materno e na amamentação. ${ }^{(26)}$ Ao profissional de saúde cabe a inserção da família no cuidado pré-natal, o que pode contribuir para a manutenção ou fortalecimento de laços desta com a gestante.

Com relação ao título Risco de Gestação, Não Intencional, foi descrito nos dois primeiros trimestres gestacionais. A gravidez não planejada tem sido investigada globalmente. No Brasil, pesquisa realizada com amplitude nacional, mostrou taxa de 55,4\% de mulheres que referiam gravidez não planejada. ${ }^{(27)}$ Nos Estados Unidos da América a gravidez não planejada parece estar em declínio: entre 2008 e 2011 as taxas foram 51\% e 45\%, respectivamente, mas manteve-se como evento frequente. ${ }^{(28)}$ Assim, parece ser justificada a ocorrência deste título de risco.

Sobre os títulos Ansiedade e Medo, elencados no segundo e terceiro trimestres, podem guardar relação com a proximidade do parto. $\mathrm{O}$ período pré-natal deve ser entendido como propício para o desenvolvimento de atividades educativas que possam contribuir com o preparo físico e emocional da gestante para o momento do parto e nascimento. ${ }^{(29)}$

Por fim, destaca-se que as gestantes incluídas no presente estudo, de baixo risco e atendidas em serviços públicos de atenção primária, tem perfil semelhante a demanda atendida por enfermeiros em muitos municípios brasileiros e, assim, os resultados poderão ser amplamente utilizados. Para futuros estudos, sugere-se investigaçóes utilizando a CIPE ${ }^{\circ}$ à abordagem das intervençóes de enfermagem, partindo destes diagnósticos identificados.

\section{Conclusão}

Os diagnósticos de enfermagem da $\mathrm{CIPE}^{\bullet}$ identificados em consulta pré-natal na atenção primária variaram pouco de acordo com o trimestre gestacional. Foram mais frequentes os diagnósticos relacionados às necessidades psicobiológicas, em comparação às psicossociais, destacando-se numericamente os diagnósticos com foco no problema. Na gravidez de risco habitual, os diagnósticos de enfermagem descreveram necessidades de nutrição, hidratação, eliminações, terapêutica e exercícios e atividades físicas. Assim, em resposta síntese, a maior parte do conjunto de diagnósticos propostos está no contexto do desenvolvimento de hábitos de vida saudáveis. Porém, nesse processo, há que se considerar a necessidade de ampliar a abordagem da gestante, de forma a contemplar títulos de diagnósticos psicossociais e psicoespirituais.

\section{Colaborações}

Costa ER, Pina MM, Jensen R, Jamas MT e Parada CMGL declaram que contribuíram com a concepção do estudo, análise e interpretação dos dados, redação do artigo, revisão crítica relevante do conteúdo intelectual e aprovação da versão final a ser publicada.

\section{Referências}

1. Penedo RM, Spiri WC. Systematization of nursing care for nurse managers. Acta Paul Enferm. 2014;27(1):86-92.

2. Azevedo OA, Guedes ES, Araújo SA, Maia MM, Cruz DA. Documentation of the nursing process in public health institutions. Rev Esc Enferm USP. 2019;53:e03471.

3. Conselho Federal de Enfermagem (COFEN). Resolução COFEN № 358/2009, de 15 de outubro de 2009 [Internet]. Dispõe sobre a Sistematização da Assistência de Enfermagem em ambientes públicos ou privados, em que ocorre o cuidado profissional de Enfermagem e dá outras providências. Brasília (DF): COFEN; 2009 [citado 2020 Mar 18]. Disponível em: http://www.cofen.gov.br/resoluocofen-3582009_4384.html

4. Garcia TR, Bartz CC, Coenen A. CIPE ${ }^{\circledR}$ : uma linguagem padronizada para a prática profissional. Garcia TR, organizador. Classificação Internacional para a Prática de Enfermagem - CIPE ${ }^{\circledR}$ : aplicação à realidade brasileira. Porto Alegre: Artmed; 2015. p. 24-36. 
5. International Organization for Standardization (ISO). Health informatics: categorial structures for representation of nursing diagnoses and nursing actions in terminological systems (ISO/FDIS 18104: 2014). Geneva: ISO; 2014. [cited 2020 May 28]. Available from: https://www. iso.org/obp/ui/\#iso:std:iso:18104:ed-2:v1:en:sec:A

6. Félix ND, Ramos NM, Nascimento MN, Moreira TM, Oliveira CJ. Nursing diagnoses from ICNP ${ }^{\circledR}$ for people with metabolic syndrome. Rev Bras Enferm. 2018;71 Suppl 1:467-74.

7. de Souza Neto VL, Costa RT, Costa DA, Belmiro SS, Lima MA, Silva RA. Diagnósticos da CIPE ${ }^{\circledR}$ de pessoas vivendo com AIDS e indicadores empíricos. Rev Bras Enferm. 2019;72(5):1226-34.

8. Horta WA. Processo de Enfermagem. São Paulo: EPU/EDUSP; 1979. 99p.

9. Querido DL, Christoffel MM, Nóbrega MM, Almeida VS, Andrade M, Esteves AP. Terminological subsets of the International Classification for Nursing Practice: an integrative literature review. Rev Esc Enferm USP. 2019;53:e03522.

10. Brasil. Ministério da Saúde. Portaria GM no 1.459, de 24 de junho de 2011: Institui, no âmbito do Sistema Único de Saúde (SUS), a Rede Cegonha. Brasília (DF): Ministério da Saúde; 2011 [citado 2020 Mar 18 ]. Disponível em: http://bvsms.saude.gov.br/bvs/saudelegis/ gm/2011/prt1459_24_06_2011.html

11. Garcia TR, Nóbrega MM, Cubas MR. CIPE ${ }^{\circledR}$ versão 2019/2020. Garcia TR (Org). Classificação Internacional para a Prática de Enfermagem (CIPE): versão 2019/2020. Porto Alegre: Artmed; 2020. p. 35-236.

12. Carvalho Al. Determinantes sociais, econômicos e ambientais da saúde. Fundação Oswaldo Cruz. A saúde no Brasil em 2030 prospecção estratégica do sistema de saúde brasileiro: população e perfil sanitário. Rio de Janeiro: Fiocruz/Ipea/Ministério da Saúde/ Secretaria de Assuntos Estratégicos da Presidência da República; 2013. Vol. 2, p. 19-38.

13. Kariuki L, Lambert C, Purwestri R, Biesalski HK. Trends and consequences of consumption of food and non-food items (pica) by pregnant women in Western Kenya. NFS J. 2016;5:1-4.

14. Walsh JM, McAuliffe FM. Impact of maternal nutrition on pregnancy outcome-does it matter what pregnant women eat? Best Pract Res Clin Obstet Gynaecol. 2015;29(1):63-78.

15. Marangoni F, Cetin I, Verduci E, Canzone G, Giovannini M, Scollo P, et al. Maternal diet and nutrient requirements in pregnancy and breastfeeding. An italian consensus document. Nutrients. 2016;8(10):E629.

16. Moreira LN, Barros DC, Baião MR, Cunha MB. Quando tem como comer, a gente come: fontes de informações sobre alimentação na gestação e as escolhas alimentares. Physis. 2018;28(3):e280321.
17. McParlin C, O'Donnell A, Robson SC, Beyer F, Moloney E, Bryant A, et al. Treatments for hyperemesis gravidarum and nausea and vomiting in pregnancy: a systematic review. JAMA. 2016;316(13):1392-401.

18. European Federation of Bottled Waters. Guidelines for adequate water intake: a public health rationale. Proceedings from EFBW Symposium IUNS 20th International Congress of Nutrition Granada, Spain, September 18, 2013. [cited 2020 Sep 18]. Available from: http:// www.efbw.eu/fileadmin/EFBW_GuidelinesforAdequateWaterlntake.pdf

19. García Duarte S, Ruíz Carmona M, Camacho Ávila M. Prevention of constipation during pregnancy with the hydration. Nutr Hosp. 2015;32(2 Suppl 2):10298.

20. Shi W, Xu X, Zhang Y, Guo S, Wang J, Wang J. Epidemiology and risk factors of functional constipation in pregnant women. PLOS One. 2015;10(7):e0133521.

21. Braga DP, Halpern G, Setti AS, Figueira RC, laconelli A Jr, Borges $E$ Jr. The impact of food intake and social habits on embryo quality and the likelihood of blastocyst formation. Reprod Biomed Online. 2015;31(1):30-8.

22. Garland M. Physical activity during pregnancy: a prescription for improved perinatal outcomes. J Nurse Pract. 2017;13(1):54-8.

23. Flack NA, Depledge J, Hay-Smith EJ, Stringer MD, Gray AR, Woodley SJ. A self-report questionnaire for pregnancy-related symphyseal pain. Musculoskelet Sci Pract. 2020 A;48:102151.

24. Liddle SD, Pennick V. Interventions for preventing and treating lowback and pelvic pain during pregnancy. Cochrane Database Syst Rev. 2015 Sep;9(9):CD001139.

25. Bacaro V, Benz F, Pappaccogli A, De Bartolo P, Johann AF, Palagini L, et al. Interventions for sleep problems during pregnancy: A systematic review. Sleep Med Rev. 2020;50:101234.

26. Pujól von Seehausen M, Pérez-Escamilla R, Couto de Oliveira MI, do Carmo Leal M, Siqueira Boccolini C. Social support modifies the association between pre-pregnancy body mass index and breastfeeding initiation in Brazil. PLoS One. 2020;15(5):e0233452.

27. Theme-Filha MM, Baldisserotto ML, Fraga AC, Ayers S, da Gama SG, Leal MD. Factors associated with unintended pregnancy in Brazil: cross-sectional results from the Birth in Brazil National Survey, 2011/2012. Reprod Health. 2016;13(S3 Suppl 3):118.

28. Finer LB, Zolna MR. Declines in unintended pregnancy in the United States, 2008-2011. N Engl J Med. 2016;374(9):843-52.

29. Velho MB, dos Santos EK, Collaço VS. [Natural childbirth and cesarean section: social representations of women who experienced them]. Rev Bras Enferm. 2014;67(2):282-9. Portuguese. 